
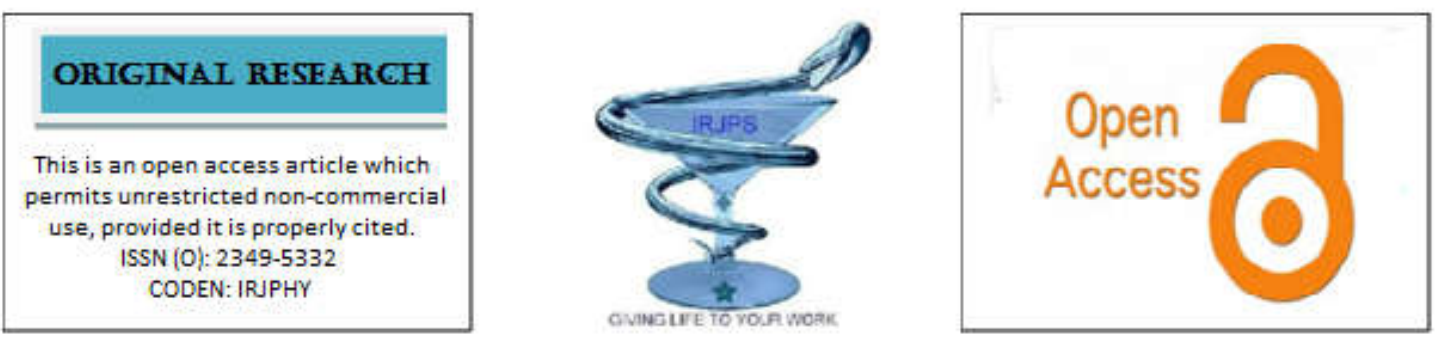

\title{
EFFECT OF OMEGA-3 FATTY ACIDS ENRICHED FISH OIL ON DEXAMETHASONE INDUCED INSULIN RESISTANCE AND HYPERTENSION IN RATS
}

\author{
Rashmi S. Chouthe ${ }^{1}$, Santosh D Shelke ${ }^{1}$, Rahul P. Kshirsagar ${ }^{2 *}$ \\ ${ }^{1}$ Department of Pharmaceutical Chemistry, Srinath College of Pharmacy, Aurangabad, Maharashtra, India \\ 431136. \\ ${ }^{2}$ Department of Pharmacology, Yash Institute of Pharmacy, Aurangabad, Maharashtra, India 431136.
}

Submitted on: 25.03.19;

Revised on: 12.04.19;

Accepted on: 18.04.19

\begin{abstract}
The role of omega - 3 fatty acids enriched fish oil ( $\omega-3 \mathrm{FA})$ in insulin resistance (IR) and its cardiovascular complications are under controversy and IR has a great significance in CVS diseases. Hence the present study was aimed to determine the effect of $\omega-3 \mathrm{FA}$ on dexamethasone induced IR and hypertension in rats. Adult male wistar rats (250-300g body weight) were divided in different groups containing six animals each. Dexamethasone was administered by injecting subcutaneously $2 \mu \mathrm{g} /$ day in divided doses twice daily for 28 days. $\omega-3$ FA $(0.5 \mathrm{ml} / \mathrm{kg} /$ day/orally) was co-administered for 28 days. Body weight, feed intake was measured daily. Measurement of systolic blood pressure was done once in week by tail cuff method. Electrocardiograph (ECG) was recorded one day before the termination of study. Biochemical parameters like glucose, insulin, triglycerides (TG), total cholesterol (TC) and MDA level were estimated in blood plasma at the end of study period. Dexamethasone treatment induced IR and hypertension. Co-administration of $\omega$-3FA significantly prevents the development of IR \& hypertension in rats. Observations of our results suggest that $\omega$-3FA might have reduced IR directly by insulin sensitizing effect in peripheral and hepatic tissues. The blood pressure lowering effect of $\omega$-3FA may be secondary to insulin sensitizing effect. In conclusion dietary supplementation of $\omega$-3FA can prevent the development of dexamethasone induced IR and hypertension.
\end{abstract}

KEY WORDS: Diabetes Mellitus, Dyslipidemia, $\omega-3$ FA, ECG, NIBP.

Corresponding Author: Rahul P Kshirsagar E-mail: rahulkshirsagar85@gmail.com Phone: +91 8790169789
Indian Research Journal of Pharmacy and Science; 20(2019)1796-1803; Journal Home Page: https://www.irjps.in DOI: 10.21276/irjps.2019.6.1.5 


\section{INTRODUCTION:}

Omega -3 fatty acids ( $\omega-3 \mathrm{FA})$ are the essential long chain polyunsaturated fatty acids. Fish oil is the major source for $\omega-3 \mathrm{FA}$ containing $\alpha$ linolenic acid, docosahexaenoic acid (DHA) and eicosapentaenoic acid (EPA) as major constituents ${ }^{1}$. Literature reveals that $\omega-3 \mathrm{FA}$ is antiarrhythmic ${ }^{2}$, antithrombotic ${ }^{3}, \quad$ antiatherosclerotic ${ }^{4}$ antihypertensive ${ }^{5}$ also found to improve endothelial function ${ }^{6}$ and lowers plasma triglycerides ${ }^{7}$.

$\omega-3 F A$ are found to be effective in reducing hyperlipidemia, hyperinsulinemia and hypertension in patients ${ }^{5,8}$. DHA is the principal component of $\omega-3 \mathrm{FA}$ responsible for their blood pressure and heart rate lowering effects ${ }^{9}$. Further, $\omega$-3FA reported to lower plasma LDL level in healthy human subjects ${ }^{10}$. Preclinical experimental studies demonstrated that dietary supplementation of $\omega-3 \mathrm{FA}$ can reduce the serum concentrations of triglycerides, total cholesterol and improves hepatic as well as peripheral insulin sensitivity ${ }^{7,11-13}$. On contrary to these beneficial effects, fish oil containing $\omega-3 \mathrm{FA}$ are reported to reverse dyslipidemia but not insulin resistance in highfructose diet induced dyslipidemia, hepatic and adipose tissue insulin resistance in humans ${ }^{14}$.

Glucocorticoids were widely used as immunosuppressive and anti-inflammatory agent, but it is accompanying with impaired insulin sensitivity and hypertension ${ }^{15}$ as a serious adverse effects. Dexamethasone without affecting insulin receptor decreases the cellular glucose uptake and alters the glucose metabolism, hence they play major role in the development of peripheral insulin resistance ${ }^{16}$. Further dexamethasone has shown cardiovascular effects such as increased mean arterial pressure (MAP), heart rate \& elevated ST segment of ECG in rats ${ }^{17}$.

Thus the present study as aimed to investigate the role of fish oil containing $\omega-3 \mathrm{FA}$ has ability to prevent dexamethasone induced insulin resistance, hypertension \& ECG changes in rats.

\section{MATERIALS AND METHODS}

Drugs and chemicals - Dexamethasone was gift from Zydus cadila Ltd. Ahmadabad. Fish Oil was gift from Merck India Ltd. Mumbai which is used as source of $\omega$-3FA [containing $17.5 \%$ Eicosapentaenoic acid (EPA) and 10.5\% Docosahexaenoic acid (DHA)]. Insulin - Radio
Immuno Assay kit was obtained from Cisbio, France. Glucose, Cholesterol, and Triglycerides kits were procured from ERBA diagnostic Mannheim Gmbh Germany.

Animals \& experimental study design Animal handling and experimental procedures were carried out in accordance with committee for the purpose of control and supervision of experiments on animal (CPCSEA) guidelines. Experimental protocol were approved by the institutional animal ethical committee of K.L.E. College of Pharmacy, Vidyanagar, Hubli (Reg. 126/1999/CPCSEA). And

Male wistar rats weighing 250-300g were purchased (National Institute of Nutrition, Hyderabad) and housed in the environmentally controlled room to temperature $23 \pm 2^{0} \mathrm{c} \&$ humidity $55^{\circ} \mathrm{c} \pm 10 \%$ in 12 -h light/dark cycle throughout the experiment. Animals had free access to water \& standard laboratory feed ad libitum.

After acclimatization period of seven days, animals were randomly selected and assigned in different groups. Each group containing six rats (n $=6)$

Group I Control: Injected with saline subcutaneously.

Group II Dexamethasone: Injected with Dexamethasone $2 \mu \mathrm{g} /$ day subcutaneously for 28 days, in divided dose $1 \mu \mathrm{g}$ twice daily ${ }^{16}$.

Group III Dexamethasone $+\omega-3$ FA: Injected with Dexamethasone $2 \mu \mathrm{g} / \mathrm{day}$ subcutaneously for 28 days, in divided dose $1 \mu \mathrm{g}$ twice daily and treated with $\omega-3 \mathrm{FA}$ enriched Fish Oil $0.5 \mathrm{ml} / \mathrm{kg} /$ day $/$ orally $^{18}$ for 28 days.

During the study period body weight, food and water intake was measured daily. Systolic blood pressure was monitored once in week by tail cuff method. Electrocardiograph (ECG) was recorded one day before and at the end of study. At the end of study blood samples were collected for biochemical investigation from overnight fasted animal through retro-orbital plexus under light ether anesthesia.

Measurement of systolic blood pressure Measurement of systolic blood pressure was done indirectly in conscious and slightly restrained, prewarmed rat by the tail cuff method (NIBP-200A, MP-35 Biopac Systems, Inc Santa Barbara, USA), an average of ten consecutive readings was noted ${ }^{19}$. Results are expressed as percentage change in 
systolic blood pressure compared to systolic blood pressure before the start of the study (0 day).

Recording of Electrocardiograph (ECG) Electrocardiograph (ECG) was recorded in anaesthetized animals (Ketamine - 50mg/kg/i.p.) using Lead I method (MP-35 Biopac Systems, Inc Santa Barbara, USA), Parameters measured were S$\mathrm{T}$ Segment Elevation \& Heart rate.

Oral glucose tolerance test - The glucose sample was administered orally $2 \mathrm{gm} / \mathrm{kg}$ (body weight) to overnight fasted experimental animals. Blood samples were collected at different time intervals as pre, 30, 60, and 120 min post- glucose load. Plasma glucose levels were measured by the glucose oxidase reaction method.

Measurement biochemical parameters Plasma insulin concentrations were measured by Immu Chem Radioimmunoassay method using RIA kit (BI-INSULIN IRMA, Cisbio, France).Total cholesterol, triglyceride and glucose levels were measured by spectrophotometer using commercially available kits according to manufacturer instructions (ERBA diagnostic Mannheim Gmbh Ltd.). Insulin resistance index was calculated by Homeostasis Model Assessment (HOMA) ${ }^{20}$ using the following formula: [insulin $(\mu \mathrm{U} / \mathrm{ml}) \times$ glucose $(\mathrm{mg} / \mathrm{dl})] / 405$.

Estimation of lipid peroxidation $(M D A)^{21}-$ Lipid peroxidation was estimated in terms of thiobarbituric acid reactive species (TBARS), using malondialdehyde (MDA) as standard. $1.0 \mathrm{ml}$ of the plasma sample was added to $2.0 \mathrm{ml}$ of the TCATBA- $\mathrm{HCl}$ reagent $(15 \% \mathrm{w} / \mathrm{v}$ TCA, $0.375 \% \mathrm{w} / \mathrm{v}$
TBA and $0.25 \mathrm{~N} \mathrm{HCl}$ ). After 15 minutes boiling content were cooled and centrifuged to remove precipitate. The absorbance was observed at 535 $\mathrm{nm}$ and concentration of MDA the samples were calculated using extinction coefficient of $1.56 \mathrm{x}$ $10^{5} \mathrm{M}^{-1} \mathrm{~cm}^{-1}$.

Statistical analysis - All the data were expressed as Mean \pm SEM. Data were subjected to One-Way analysis of variance (ANOVA) followed by Newman-Keuls Multiple Comparison test where $\mathrm{P}<0.05$ was considered as statistically significant.

\section{RESULTS}

Body weight and food intake - Significant decrease in in body weight $(\mathrm{P}<0.001)$ and cumulative food intake $(\mathrm{P}<0.05)$ were observed in dexamethasone administered rats when compared to control (Table-1). Co-administration of $\omega$-3FA had no effect on Dexamethasone induced reduction in body weight and food intake (Table-1).

Plasma Glucose, Insulin, Triglyceride and

Total cholesterol - Administration of Dexamethasone in rats lead to significant increase in plasma glucose $(\mathrm{P}<0.001)$, plasma insulin $(\mathrm{P}<0.001)$ and triglyceride level $(\mathrm{P}<0.001)$ but had no effect on total cholesterol level when compared to control (Table-1). Co-administration of $\omega$-3FA significantly prevented the dexamethasone induced changes in plasma glucose $(\mathrm{P}<0.001)$, plasma insulin $(\mathrm{P}<0.01)$, plasma triglyceride $(\mathrm{P}<0.01)$, as well as total cholesterol level $(\mathrm{P}<0.001)$ when compared to Dexamethasone treated rats (Table-1).

Table-1: Effect of $\omega$-3FA on body weight gain, cumulative food intake and biochemical parameters.

\begin{tabular}{|c|c|c|c|}
\hline 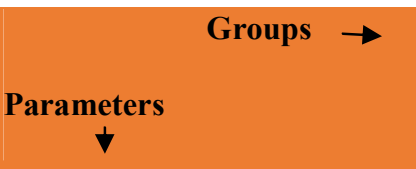 & $\begin{array}{l}\text { Control } \\
\text { (C) }\end{array}$ & $\begin{array}{l}\text { Dexamethasone } \\
\text { (D) }\end{array}$ & 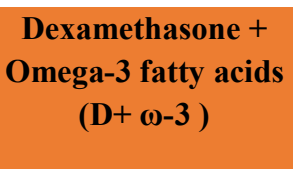 \\
\hline \% Body weight gain (gm) & $22.520 \pm 1.59$ & $2.6840 \pm 2.76^{* * *}$ & $7.1570 \pm 1.1570$ \\
\hline Cumulative food intake (gm) & $460.2 \pm 5.32$ & $422.3 \pm 18.35^{*}$ & $405.2 \pm 9.954$ \\
\hline Glucose (mg/dl) & $80.42 \pm 2.440$ & $103.0 \pm 4.16^{* * *}$ & $76.74 \pm 2.68^{\# \# \#}$ \\
\hline Triglycerides (mg/dl) & $122.5 \pm 16.53$ & $386.9 \pm 58.23 * * *$ & $164.5 \pm 33.08^{\# \#}$ \\
\hline Total Cholesterol (mg/dl) & $67.67 \pm 5.08$ & $75.03 \pm 4.09$ & $41.08 \pm 3.90^{\# \# \#}$ \\
\hline Insulin $(\mu \mathrm{U} / \mathrm{ml})$ & $0.5717 \pm 0.027$ & $1.128 \pm 0.030 * * *$ & $0.9417 \pm 0.032^{\# \#}$ \\
\hline MDA & $3507 \pm 819.2$ & $9533 \pm 2274 * * *$ & $3764 \pm 843.6^{\# \# \#}$ \\
\hline HOMA-IR & $0.1129 \pm 0.068$ & $0.2912 \pm 0.020^{* * *}$ & $0.1775 \pm 0.011^{\# \#}$ \\
\hline
\end{tabular}


Data are expressed as Mean $\pm \mathrm{SEM}, \mathrm{n}=6, * \mathrm{p}<0.05$ when compared to control, $* * \mathrm{p}<0.01$ when compared to control, $* * * \mathrm{p}<0.001$ when compared to control, ${ }^{\#} \mathrm{p}<0.05$ when compared to Dexamethasone, ${ }^{\# \#} \mathrm{p}<0.01$ when compared to Dexamethasone, ${ }^{\# \#} \mathrm{p}<0.001$ when compared to Dexamethasone, C: Control, D: Dexamethasone, $\mathrm{D}+\omega-3$ : Dexamethasone + Omega 3fatty acids.

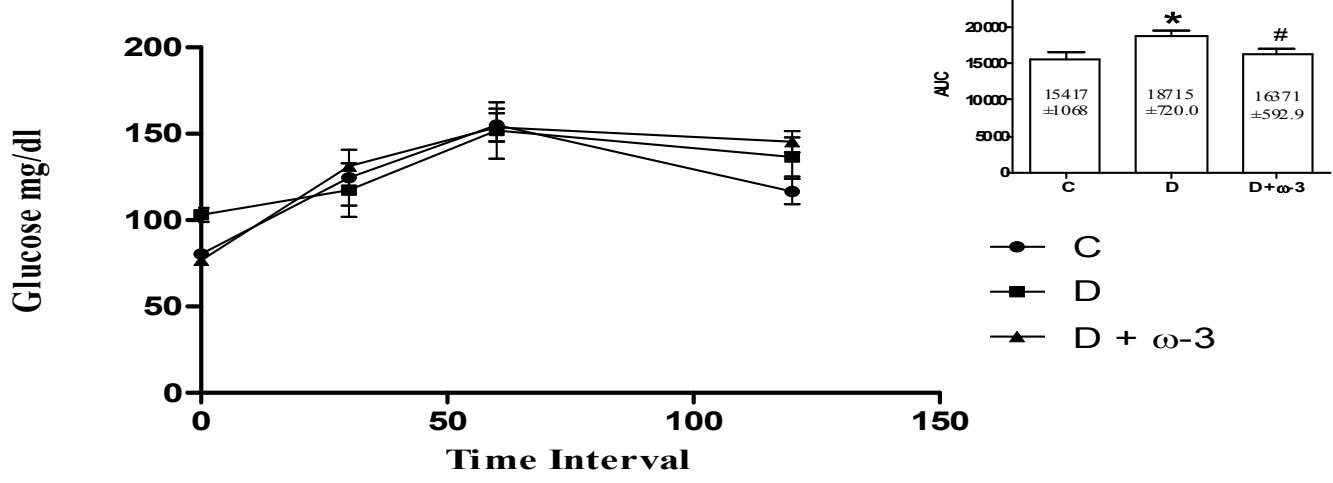

Figure-1: Effect of $\omega$-3FA on total AUC of plasma glucose after oral glucose challenge in dexamethasone treated rats.

Data are expressed as Mean $\pm \mathrm{SEM}, \mathrm{n}=6,{ }^{*} \mathrm{p}<0.05$ when compared to control, $* * \mathrm{p}<0.01$ when compared to control, $* * * \mathrm{p}<0.001$ when compared to control, ${ }^{\#} \mathrm{p}<0.05$ when compared to Dexamethasone, ${ }^{\#} \mathrm{p}<0.01$ when compared to Dexamethasone, ${ }^{\# \#} \mathrm{p}<0.001$ when compared to Dexamethasone, C: Control, D: Dexamethasone, $\mathrm{D}+\omega-3$ : Dexamethasone + Omega 3 fatty acids.

Insulin resistance index (HOMA-IR) Significant increase in HOMA-IR index in Dexamethasone treated rats lead to development of insulin resistance $(\mathrm{P}<0.001)$ as compared to control animals (Table-1). Co-administration of $\omega$-3FA in Dexamethasone administered rats significantly decreased insulin resistance index $(\mathrm{P}<0.001)$ when compared to Dexamethasone administered rats (Table-1).

Oral Glucose Tolerance Test (OGTT) Significant increase in total AUC of plasma glucose $(\mathrm{P}<0.05)$ during $120 \mathrm{~min}$ indicates development of glucose intolerance in Dexamethasone treated rats when compared to control (Figure-1). Coadministration of $\omega$-3FA in Dexamethasone administered rats significantly improved the glucose tolerance $(\mathrm{P}<0.05)$ when compared to Dexamethasone treated rats (Figure-1).
Lipid Peroxidation (plasma MDA level) Significant increase in plasma MDA level $(\mathrm{P}<0.001)$ were observed in Dexamethasone treated rats as compared to control (Table-1). Coadministration of $\omega-3 \mathrm{FA}$ in Dexamethasone administered rats significantly decreased plasma MDA level $(\mathrm{P}<0.001)$ when compared to Dexamethasone administered rats (Table-1).

Systolic Blood pressure - The significant elevation of systolic blood pressure when compared to control $(\mathrm{P}<0.001)$ (Figure-2) was observed with dexamethasone. The increase in systolic blood pressure was significantly high from $7^{\text {th }}$ day and continued to increase till the end of the study. Coadministration of $\omega$-3FA partially but significantly reduces the elevated systolic blood pressure due to dexamethasone from $14^{\text {th }}$ day $(\mathrm{P}<0.001)$ onwards till the end of study (Figure - 2). 


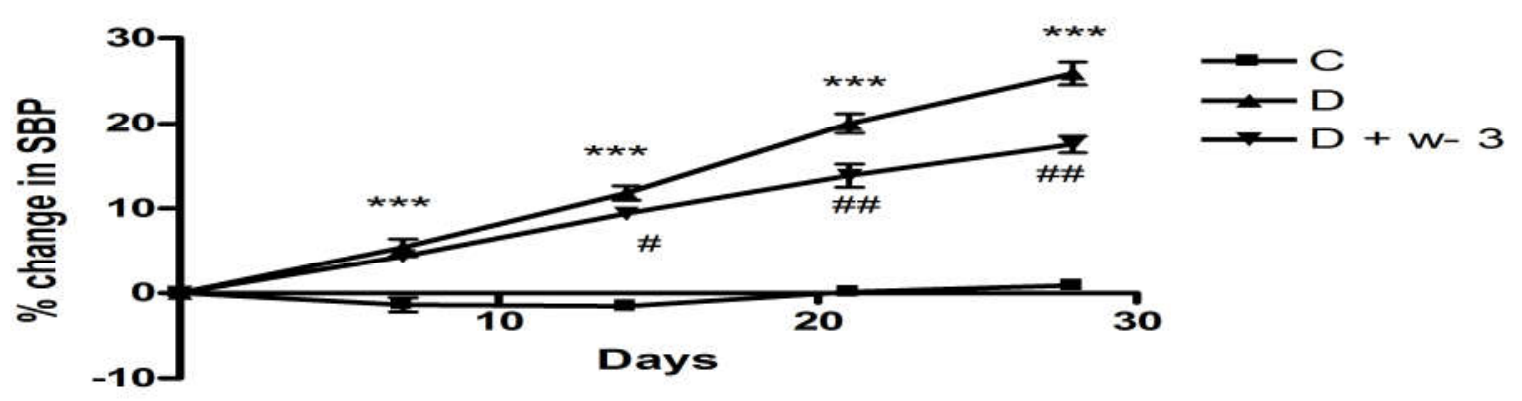

Figure-2: Effect of $\omega$-3FA on \% change in systolic blood pressure.

Data are expressed as Mean \pm SEM, $n=6, * p<0.05$ when compared to control, ${ }^{*} * \mathrm{p}<0.01$ when compared to control, $* * * p<0.001$ when compared to control, ${ }^{\#} \mathrm{p}<0.05$ when compared to Dexamethasone, ${ }^{\# \#} \mathrm{p}<0.01$ when compared to Dexamethasone, ${ }^{\# \#} \mathrm{p}<0.001$ when compared to Dexamethasone, C: Control, D: Dexamethasone, $\mathrm{D}+\omega-3$ : Dexamethasone + Omega 3 fatty acids.

Electrocardiograph - Dexamethasone showed significant elevation of ST-segment $(\mathrm{P}<0.001)$ \& heart rate $(\mathrm{P}<0.01)$ when compared to control. Prototype ECG showed the changes in ST- segment (Figure-3). Co-administration of $\omega$-3FA in Dexamethasone administered rats significantly prevented the elevation of ST-segment $(\mathrm{P}<0.001) \&$ heart rate $(\mathrm{P}<0.01)$ when compared to Dexamethasone treated rats (Figure-3).

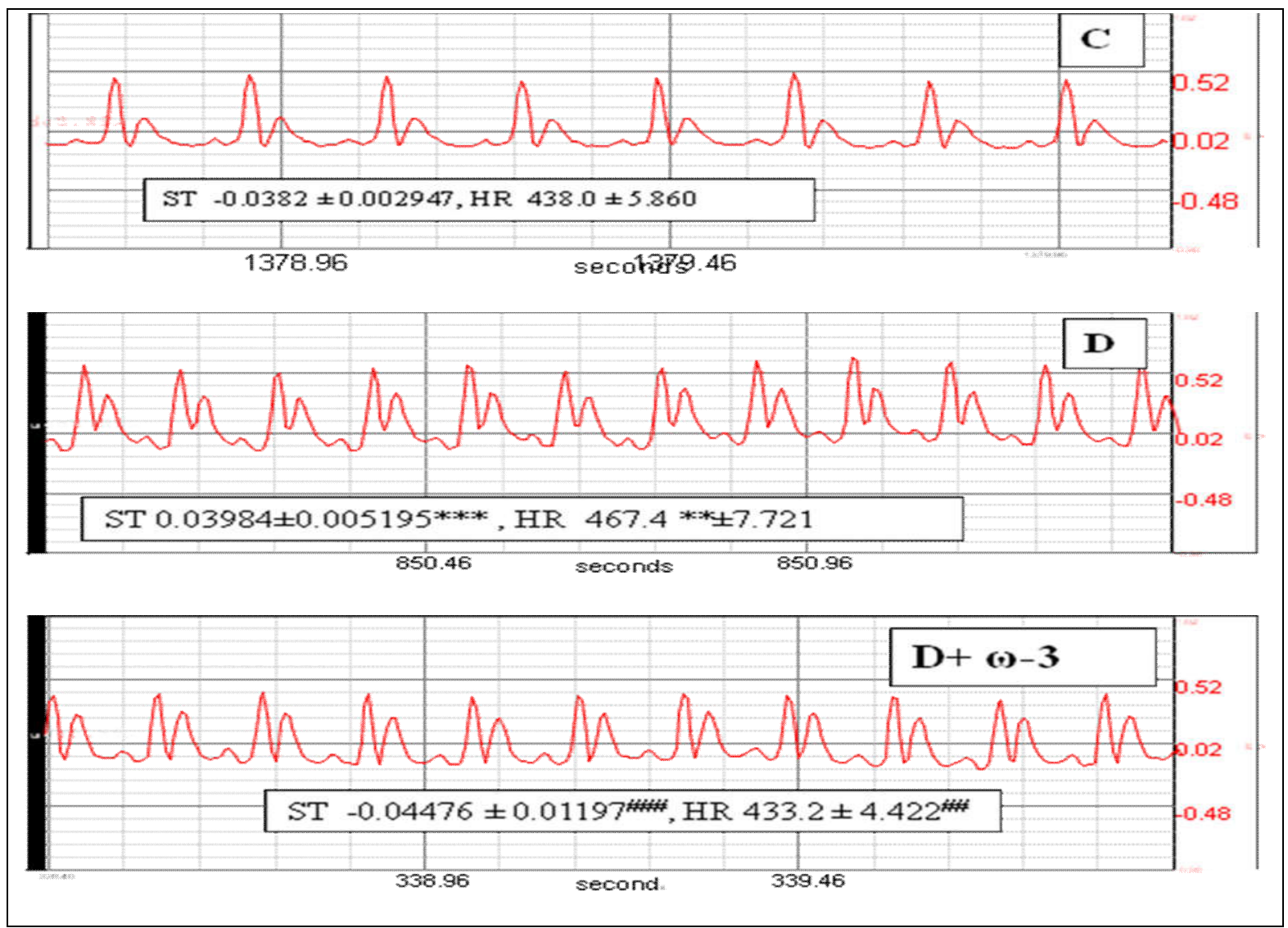

Figure-3: Effect of $\omega$-3FA on ECG (ST - Segment Elevation \& Heart rate). 
Data are expressed as Mean $\pm \mathrm{SEM}, \mathrm{n}=6,{ }^{*} \mathrm{p}<0.05$ when compared to control, $* * \mathrm{p}<0.01$ when compared to control, ***p $<0.001$ when compared to control, ${ }^{\#} \mathrm{p}<0.05$ when compared to Dexamethasone, ${ }^{\#} \mathrm{p}<0.01$ when compared to Dexamethasone, ${ }^{\# \#} \mathrm{p}<0.001$ when compared to Dexamethasone, C: Control, D: Dexamethasone, $\mathrm{D}+\omega-3$ : Dexamethasone + Omega 3 fatty acids.

\section{DISCUSSION}

The objective of the present study was to explore the ability of $\omega$-3FA enriched fish oil to prevent dexamethasone induced insulin resistance \& hypertension. Results of the present study demonstrate that dietary supplementation of $\omega$-3FA enriched fish oil can prevent the dexamethasone induced metabolic abnormalities, insulin resistance, glucose intolerance, systolic blood pressure \& elevation of ST segment of ECG.

Fish oil rich in long chain n-3 polyunsaturated fatty acids ( $\omega-3 \mathrm{FA})$ containing EPA \& DHA has been reported to exert beneficial metabolic \& cardiac effects in clinical and animal studies $^{1-8}$. A recent report shows its beneficial effects on insulin resistance and glucose intolerance in rat model of dietary - induced obesity. In this dietary model, insulin resistance is mainly mediated through abnormal lipid metabolism especially triglyceride metabolism. Increased triglyceride is known to interfere with glucose uptake by decreased translocation of GLUT4 $4^{11}$. Insulin resistance can also be induced by certain drugs like glucocorticoids such as dexamethasone, as an adverse effect, where they directly interfere with GLUT4 translocation ${ }^{22}$.

Consistent with previous reports, administration of dexamethasone for 4 week leads to development of insulin resistance characterized by hyperglycemia, hyperinsulinemia, glucose intolerance $^{16}$. In the present study co-administration of $\omega-3 \mathrm{FA}$ enriched fish oil significantly prevented these changes. Previous study has reported that fish oil enhances insulin signaling by increasing Akt phosphorylation ${ }^{11}$. Further it has also been reported to reduce hepatic glucose production ${ }^{9}$. Based on these reports \& observations from our results, $\omega$ 3FA enriched fish oil might have reduced dexamethasone induced insulin resistance directly

\section{REFERENCES}

1. Puneet K, Timothy W, Gregory YH, New Approaches to Therapy with Omega - 3 by insulin sensitizing effect on peripheral and hepatic tissues.

Dexamethasone administration is known to elevate systolic blood pressure in experimental animals along with insulin resistance ${ }^{23}$. Increasing number of evidence suggest that hyperinsulinemia and hypertension are causally related for each other. In the present study fish oil significantly reduced the systolic blood pressure; along with reduction in plasma insulin. This observation indicates that the blood pressure lowering effect of fish oil may be secondary to insulin sensitizing effect. Further, supplementation of $\omega$-3FA enriched fish oil prevented dexamethasone induced elevation of ST segment \& heart rate, hence $\omega$-3FA enriched fish oil supplementation has significant potential in preventing dexamethasone induced cardiovascular events.

Oxidative stress has been attributed to play a significant role in various diseases including insulin resistance and cardiovascular diseases. Consistence with this notion, dexamethasone administration is associated with systemic oxidative stress. In the present study supplementation of $\omega$ 3FA enriched fish oil significantly reduced the oxidative stress. These observations suggest that beneficial effects of $\omega$-3FA may be partially due to antioxidant nature of fish oil. However, this notion needs further study.

In conclusion, dietary supplementation of omega-3 fatty acids enriched fish oil can prevent the development of dexamethasone induced insulin resistance \& hypertension. Implicating the significant role in managing the drug induced adverse effect.

\section{ACKNOWLEDGEMENTS}

The authors would like to thank Dr. B. M. Patil, Principal, KLES's college of Pharmacy for their support in providing research facilities.

Fatty Acids, Current Atherosclerosis Reports, 2008; 10(1):79-87.

2. Leaf A, Kang JX, Xiao YF, Billman GE, Clinical prevention of sudden cardiac 
death by $n-3$ polyunsaturated fatty acids and mechanism of prevention of arrhythmias by n-3 fish oils, Circulation, 2003; 107(21):2646-2652.

3. Kristensen SD, Iversen AM, Schmidt EB, n-3 polyunsaturated fatty acids and coronary thrombosis, Lipids, 2001;36 Suppl:S79-82.

4. Thies F, Garry JM, Yaqoob P, Rerkasem K, Williams J, Shearman CP, Association of n-3 polyunsaturated fatty acids with stability of atherosclerotic plaques: a randomised controlled trial, Lancet, 2003 Feb 8;361(9356):477-85.

5. Morris MC, Sacks F, Rosner B, Does Fish Oil Lower Blood Pressure? A MetaAnalysis of Controlled Trials, Circulation, 1993; 88:523-533.

6. Khan F, Elherik K, Bolton-Smith C, Barr R, Hill A, The effects of dietary fatty acid supplementation on endothelial function and vascular tone in healthy subjects, Cardiovasc Res, 2003 Oct 1;59(4):955-62.

7. Kobatake $\mathrm{Y}$, Hirahara $\mathrm{F}$, Innami $\mathrm{S}$, Nishide E, Dietary effect of omega-3 type polyunsaturated fatty acids on serum and liver lipid levels in rats, J Nutr Sci Vitaminol (Tokyo) 1983 Feb;29(1):11-21.

8. Yam D, Friedman J, Bott-Kanner G, Omega-3 fatty acids reduce hyperlipidaemia, hyperinsulinaemia and hypertension in cardiovascular patients, Journal of Clinical \& Basic Cardiology, 2002; 5 (3), 229-231.

9. Mori TA, Bao DQ, Burke V, Puddey IB, Beilin LJ, Docosahexanoic Acid but Not Eicosapentaenoic acid Lowers Ambulatory Blood Pressure and Heart Rate in Humans, Hypertension, 1999;34:253-260.

10. Illingworth DR, Harris WS, Connor WE, Inhibition of low density lipoprotein synthesis by dietary omega-3 fatty acids in humans, Arteriosclerosis, 1984 MayJun;4(3):270-5.

11. Neschen S, Morino K, Dong J, WangFischer Y, Cline GW, Romanelli AJ, n-3 Fatty Acids Preserve Insulin Sensitivity In Vivo in a Peroxisome Proliferator Activated Receptor $-\alpha$ - Dependent Manner, Diabetes, 2007; 56:1034-1041,.
12. Ghafoorunissa, Ibrahim A, Rajkumar L, Acharya V, Dietary(n-3) Long Chain Polyunsaturated Fatty Acids Prevent Sucrose- Induced Insulin Resistance in Rats, J Nutri, 2005 Nov;135(11):2634-8.

13. Samane S, Christon R, Dombrowski L, Turcotte S, Charrouf Z, Lavigne C, Fish oil and argan oil intake differently insulin resistance and glucose intolerance in a rat model of dietary-induced obesity, Metabolism, 2009 Jul;58(7):909-19.

14. Faeh D, Minehira K, Schwarz JM, Periasamy R, Park S, Tappy L, Effect of fructose overfeeding and fish oil administration on hepatic de novo lipogenesis and insulin sensitivity in healthy men, Diabetes, 2005 Jul; 54(7): 1907-1913..

15. Qi D, Pulinilkunnil T, An D, Ghosh S, Abrahani A, Pospisilik JA, Single-Dose Dexamethasone Induces Whole-Body Insulin Resistance and Alters Both Cardiac Fatty Acid and Carbohydrate Metabolism, Diabetes, 2004 Jul; 53(7): 1790-1797. .

16. Severino C, Brizzi P, Solinas A, Secchi G, Maioli M, Tonolo G, Low-dose dexamethasone in the rat: a model to study insulin resistance, Am J Physiol Endocrinol Metab, 2002; 283: E367-E373,

17. Rajashree S, Puvanakrishnan R, Dexamethasone induced alteration in enzymatic and nonenzymatic antioxidant status in heart and kidney of rats, Mol Cell Biochem, 1998; 181(1-2):77-85.

18. Chitra S, Semmalar R, Shyamala Devi CS, Effect of fish oil on cigarette smoking induced dyslipidemia in rats, Indian $\mathrm{J}$ of Pharmacology, 2000; 32: 114-119.

19. Buñag RD, Butterfield J, Tail-cuff blood pressure measurement without external preheating in awake rats, Hypertension, 1982; 4(6):898-903.

20. Henry RR, Insulin Resistance: From Predisposing Factor to Therapeutic Target in Type 2 Diabetes, Clin Ther, 2003;25 Suppl B:B47-63.

21. Ray A, Chaudhri SR, Majumdar B, Bandyopadhyay SK, Antioxidant activity of ethanol extract of rhizome of Picrorrhiza kurroa on indomethacin 
induced gastric ulcer during healing, Indian J Clin Biochem, 2002, 17 (2) 44-51.

22. Weinstein SP, Wilson CM, Pritsker A, Cushman SW, Dexamethasone inhibitis insuin-stimulated recruitment of GLUT 4 to the cell surface in a rat skeletal muscle, Metabolism, 1998 Jan;47(1):3-6.
23. Tonolo G, Fraser R, Connell JM, Kenyon CJ, Chronic low-dose infusion of dexamethasone in rats: effects on blood pressure body weight and plasma atrial natriuretic peptide, J Hypertens, 1988 Jan;6(1):25-31. 